betreuten Gefangenen entlassen sind. Es ist wichtig, daß sie in eine Knastkommune ziehen. Eine Knastkommune besteht aus ehemaligen Häflingen und $\mathrm{SH}-$ Genossen. Durch den gemeinsamen Lebensvollzug ergeben sich ständige Diskussionen, von denen alle in der Knastkommune profitieren. Außerdem ist die Mitarbeit der Häftlinge unheimlich wichtig, weil sie am besten wissen, was im Knast getan werden muß und was man lieber weglassen sollte, damit die Gefangenen nicht zu starken Repressalien ausgesetzt sind.

Dies alles tun wir nicht als karitative Organisation. Wir haben erkannt, daß karitative Arbeit verschleiert, mildert, das System stabilisiert, daß die Widersprüche aufgedeckt und verstärkt werden müssen. Wir haben erkannt, daß wir das Lumpenproletariat organisieren müssen, damit die Herrschenden es nicht gegen uns organisieren. Deshalb beschränkt sich unsere Arbeit nicht auf den Knast, wir bereiten Stadtteilarbeit in einer Randgruppensiedlung vor. Wir werden noch mehr machen. Um zu einer politischen Kraft zu werden, müssen die Randgruppen in allen Lebensbereichen für ihre Interessen kämpfen.

Die SH versteht sich als Bestandteil der internationalen Befreiungsfront gegen jede Unterdrückung des Menschen durch Menschen. Wir wollen keine Massenpartei gründen, sondern wir wollen politische Aktionen vermitteln und vorbereiten. Noch können wir legal arbeiten, aber die Reaktion der Herrschenden schläft nicht. Unsere Wohnungen werden durchsucht, unsere Telefone abgehört, einige von uns wurden verhaftet, aber:

Wir kämpfen für das Volk! Wir werden siegen!

Es gibt bereits Schwarze Hilfen in Westdeutschland: SH-Köln, SH-Hamburg, SH-Hannover, SH-Wolfsburg, SH-Mainz, SH-München

Wir brauchen praktische Solidarität, d. h. Information von Gefangenen, Bücher, Zeitschriften und Geld.

Schwarze Hilfe Westberlin* Verantwortlich: Inge Viett

\title{
Ein Angriff auf die Freiheit von Forschung und Lehre
}

\section{Der $\gg$ Fall Seifert $\propto$ und sein Kontext}

Am I9. Januar 1972 behauptete der in Düsseldorf als Mitglied der sogenannten Baader-Meinhof-Gruppe angeklagte Karl-Heinz Ruhland, der hannoversche Professor Peter Brückner habe Mitglieder der Gruppe beherbergt. Der niedersächsische Kultusminister verbor Brückner daraufhin die Führung seiner Dienstgeschäfte $\left(\$ 6_{7} \mathrm{Nds}\right.$. Beamtengesetz, entsprechend $\$ 60$ Bundesbeamtengesetz). Aus Protest gegen die Suspendierung Brückners fand am 25. Januar 1972 in der TU Hannover ein teach-in statt. In dieser Veranstaltung hielt Jürgen Seifert, Professor für Wissenschaft von der Politik in Hannover, eine Rede, ${ }^{1}$ in der er u. a. die Suspendierung Brückners kritisierte und davor

\footnotetext{
* Kontakradresse: Inge Vietr, I Berlin 30, Postfach 430I; Spendenkonto: Berliner Diskonto Bank, Berlin 65, Konto Nr. 240/5389, SH-Sonderkonto Viett

1 Abgedrudst u. 2. in der Deutschlandausgabe der Frankfurter Rundschau vom 10. 2. 1972; sowie in einer beim Sozialistischen Büro, 60s Offenbach, Postfach 59I, erhältlichen Dokumenration (Preis 0,50 DM).
} 
warnte, im Zusammenhang mit der Verfolgung der sogenannten Baader-Meinhof-Gruppe rechtsstaatliche Prinzipien zu verletzen. Wegen seiner Rede wurde Seifert in der konservativen und zum Teil auch in der liberalen Presse scharf kritisiert. Der niedersächsische Kultusminister leitete am 26. Januar 1972 gegen Seifert die Vorermittlungen für ein Disziplinarverfahren ein. Ihm wurde vorgeworfen, er habe bei seiner Rede auf dem teach-in diejenige Mäßigung und $\mathrm{Zu-}$ rückhaltung nicht gewahrt, die sich aus der Stellung eines Beamten gegenüber der Gesamtheit und aus der Rücksicht auf die Pflichten seines Amtes ergeben ( $\$ 6 \mathrm{I}$ Abs. 3 Nds. Beamtengesetz, entsprechend $\$ 53$ Bundesbeamtengesetz).

Die Fälle Brückner und Seifert, die Kontroverse um den Spiegel-Artikel Heinrich Bölls vom IO. Januar I972, die Presseberichterstattung über den RuhlandProzeß und über die Fahndung nach der sogenannten Baader-Meinhof-Gruppe? stehen im Zusammenhang mit dem Versuch zu einer umfassenden Gegenreformkampagne $e^{3}$ in der Bundesrepublik. Die Kampagne verfolgt einen doppelten Zweck: Sie will die seit der Studentenrevolte in der Bundesrepublik neu entstandene Linke diffamieren und sie seit dieser Zeit in Gang gekommenen Reformprozesse rüdkgängig machen. Das Hauptinstrumentarium der Gegenreformkampagne ist leicht $\mathrm{zu}$ durchschauen: Linke und Liberale sollen politisch neutralisiert werden, indem sie mit (angeblich) Kriminellen gleichgesetzt werden. Nachdem die frühere Gleichsetzung mit »den Kommunisten « nicht mehr wirkt, mußte ein neuer Aufhänger für die politische Diffamierung von Linken und Liberalen ${ }^{4}$ gefunden werden: dazu bot sich die sogenannte Baader-Meinhof-Gruppe an.

Im Folgenden wird an einem Einzelfall untersucht, was eine Gegenreform für die Hochschule bedeuten könnte. Die Betrachtung ist überwiegend juristisch. Verschiebungen in der rechtlichen Beurteilung zeigen aber sehr deutlich, in welcher Richtung die politische Entwicklung verlaufen kann.

\section{Uneinschränkbarkeit der Freibeit von Forschung und Lebre}

Das Ministerium ist bei der Anordnung der Vorermittlungen gegen Jürgen Seifert zu Unrecht davon ausgegangen, daß die Vorschrift des Beamtenrechts, die den Beamten zu politischer Mäßigung verpflichtet, auf den vorliegenden Fall anwendbar ist. Denn Jürgen Seifert hat mit seiner Rede am 25. Januar 1972 von dem ihm in Art. 5 Abs. 3 GG zugewiesenen Recht auf Freihcit von Wissenschaft, Forschung und Lehre Gebrauch gemacht. Dieses Grundrecht unterliegt im Gegensatz zu dem Recht auf freie Meinungsäußerung nicht den Schranken der allgemeinen Gesetze. ${ }^{5}$ Das heißt, die Freiheit von Wissenschaft und Lehre darf nicht durch Vorschriften eingeengt werden, die darauf abzielen, gerade die Ausübung dieses Grundrechtes selbst einzuschränken. Der Bonner Ministerialrat Dallinger drückt das so aus: »Zusammenfassend ist festzustellen, daß das Freiheits-

2 Vgl. dazu Redaktion der Kritischen Justiz >Mit allen Mittelne, KJ I97I, S. 106; Peter Brüdkner, Marginalien zur Interpretation einer Campagne*, KJ I97I, S. 108; die Dokumentation des Sozialistischen Anwaltkollektivs Westberlin Autoritäre Politisierung der Strafjustiz*, KJ I97I, S. 40I; sowie $\gg$ Die objektiven Grundlagen der zweiten Verfolgungswelle*, Rote Robe I/72.

3 Dazu ausführlich: Michael Vester, $\gg$ Dic Gegenreform-Kampagne 1970-1972 - Zur Einordnung der, Fällec Brüdkner und Seifert*, in: links, Sozialistische Zeitung, März 1972.

4 Zur politischen Diffamierung in den fünfziger Jahren vgl. Heinrich Hannover, Politische Diffamierung der Opposition, Dortmund r 962.

$\checkmark$ Entspr. für die Kunstfreiheit BVerfGE $30,173,193$. 
recht aus Art. 5 Abs. 3 jede Bindung hinsichtlich des Inhalts von Forschung und Lehre ausschließt; davon sind insbesondere umfaßt die vertretene Lehrmeinung, das Forschungsergebnis und seine Verbreitung, die gewählte Methode, die Art der Darstellung und der wissenschaftliche Ansatz. " ${ }^{\theta}$ Eine inhaltliche Bindung von Forschung und Lehre wäre es aber, wenn ein Hochschulangehöriger bei der Ausïbung seines Grundrechtes nach Art. 5 Abs. 3 GG einer beamtenrechtlichen Pflicht zur politischen Mäßigung und Zurückhaltung unterläge.

$\mathrm{Daß}$ die Hochschullehrer bei der Ausübung ihres Berufs eine weitgehende Unabhängigkeit haben, war bisher unbestritten. ${ }^{7}$ Der Dienststrafhof für die Länder Niedersachsen und Schleswig-Holstein hat den beamtenrechtlichen Sonderstatus des Hochschullehrers bereits 1957 ausdrüdklich anerkannt. ${ }^{8}$.

Soweit ein Beamter unmittelbar in die staatliche Hierarchie eingeordnet ist, muß er zu der seinem Amte angemessenen politischen Loyalität verpflichtet werden können. Hochschulangehörige sind aber, wie sich auch aus Art. 5 Abs. 3 GG ergibt, nicht in die staatliche Hierarchie eingegliedert. Sie sind bei der Ausübung von Forschung und Lehre unabhängig von staatlichen Weisungen. Damit ergibt sich, daß eine Vorschrift wie $\ 6_{\mathrm{I}}$ Abs. 3 Nds. Beamtengesetz, die die politische Loyalität des Beamtenkorps sicherstellen soll, für Hochschulangehörige bei der Ausübung ihrer Rechte nach Art. 5 Abs. 3 GG nicht gilt.

\section{Die Geltung von Art. S Abs. 3 GG für den teach-in Beitrag}

Mit seiner Rede vom 25. Januar 1972 hat Jürgen Seifert von seinem Recht auf Freiheit von Wissenschaft und Forschung Gebrauch gemacht. Als Professor für Wissenschaft von der Politik ist es seine dienstliche Aufgabe, die aktuelle politische Entwicklung zu analysieren. Dabei ist es auch seine Pflicht, die Tagesereignisse in größere Zusammenhänge zu stellen und auf Widersprüche und Versäumnisse hinzuweisen. ${ }^{\ominus}$ Gerade das ist, wenn auch in der einem teach-in angepaßten zugespitzten Form, der Inhalt seiner Rede.

Wenn Seifert die Ergebnisse seiner Uberlegungen zum Ruhland-Prozeß und zum Fall Brückner einem größeren universitären Publikum in den Räumen der Hochschule vortrug, dann hat er außerdem von seinem Recht auf Freiheit der Lehre Gebrauch gemacht. Es handelte sich bei dem teach-in nicht um eine beliebige politische Veranstaltung, wie etwa eine Parteiversammlung, sondern um ein universitätsinternes Ereignis. Das Grundrecht auf Freiheit der Lehre gilt nicht nur für traditionelle Universitätsveranstaltungen wie Vorlesungen und Seminare, sondern es schützt auch Hochschulveranstaltungen neuen Typs wie das erst in den letzten Jahren aufgekommene teach-in. Ein teach-in ist für die Wahrnehmung der Lehrfreiheit besonders geeignet, weil hier verhältnismäßig viele Studenten zusammenkommen, bei denen für das behandelte Thema besonderes Interesse und zum Teil auch Sachkunde vorausgesetzt werden kann. Die Beteiligung an einer solchen Veranstaltung ist für einen Hochschullehrer, der den Kontakt zu seinen Studenten nicht verlieren will, geradezu notwendig.

$\mathrm{Da}$ das teach-in unter den universitären Veranstaltungen eine Sonderstellung einnimmt, ist es verständlich, daß dort eine andere Sprache gesprochen werden

\footnotetext{
- Peter Dallinger, $\$$ Wissenschaftsfreiheit und Mitbestimmung*, JZ 1971, S. 665, 667.

7 Vgl. BVerfGE 3,58, I 5 I.

8 Amtliche Sammlung der Entscheidungen des OVG Lüneburg, Band II, S. $474 \mathrm{ff}$.

- Eine Pflicht zur politischen Kritik von Hochschullehrern bejaht auch: H. J. Wolff, Verwaltungsrecht II, 2. Aufl., München und Berlin 1967, $\$$ II4 IV a, S. 434 .
} 
muß als etwa bei einer Antrittsvorlesung. Die Größe des Auditoriums und die begrenzte Redezeit machen rhetorische Zuspitzungen unvermeidlich. Dies gilt umso mehr, als das teach-in vom 25. Januar I 972 der Analyse eines die Universität berührenden Ereignisses galt, das bei der Mehrzahl der Anwesenden große Unruhe ausgelöst hatte. Die spezifische Form des teach-in Beitrages schließt also die Berufung auf die Lehrfreiheit nicht aus.

\section{Treue zur Verfassung}

Die einzige Schranke, der die Freiheit von Wissenschaft, Forschung und Lehre unterliegt, ist die Treue zur Verfassung (Art. 5 Abs. 3 S. 2 GG). Der niedersächsische Ministerpräsident Kubel hat die Auffassung vertreten, Seifert habe mit seiner Rede gegen seine Treuepflichten als Landesbeamter verstoßen. Daß Seifert eine politische Treuepflicht gegenüber der Landesregierung bei der Ausübung seines Grundrechtes nach Art. 5 Abs. 3 GG nicht hat, wurde schon festgestellt. Kubel könnte jedoch auch gemeint haben, die Rede verstoße gegen das Gebot zur Treue gegenüber dem Grundgesetz i. S. des Art. s Abs. 3 S. 2 GG.

Daß die Treue zur Verfassung Kritik an aktuellen politischen Entwicklungen nicht ausschließt, war schon immer herrschende Meinung. Zulässig ist sogar Kritik am Grundgesetz. ${ }^{10}$

Wie weit gerade in Niedersachsen der Spielraum für Kritik am Grundgesetz reicht, zeigt das Beispiel des Göttinger Ordinarius Werner Weber. Ohne daß bisher irgendjemand auf die (in der Tat verfehlte) Idee gekommen wäre, ihn dafür dienstrechtlich zur Verantwortung zu ziehen, kritisiert Werner Weber die rechtsstaatliche Ordnung des Grundgesetzes: »... die unbändige vitale Kraft des Politischen, der man in Weimar nicht ausgewichen war, erscheint im Grundgesetz als überlistet. «11 „Das vollendete justizstaatliche Idealsystem des Bonner Grundgesetzes aber droht Verwaltung, Regierung und Gesetzgebung ganz in die Umzingelung durch Verfassungs-, Verwaltungs- und Zivilgerichte zu verstricken. «12 Weber vermißt im Grundgesetz eine dem Art. 48 WRV entsprechende Regelung: »Bei den Verfassungsschöpfern von Bonn überwog die Furcht vor so viel Kühnheit, die Ablehnung starker staatlicher Kraftentfaltung und die Sorge um den monopolistischen Einfluß der Parteiorganisationen. Sie haben zwar deshalb nicht die Notwendigkeit von Ausnahmebefugnissen gänzlich ignoriert, aber die kraftvolle Lösung des Art. 48 WRV zu einem blassen Abbild verdünnt. «13

Das Verwaltungsgericht Berlin hat kürzlich festgestellt, was für einen Verstoß gegen die Treueklausel des Art. 5 Abs. 3 S. 2 GG erforderlich ist: »... eine aktive politische Betätigung, eine aufrufende, aggressive Äußerung verfassungsfeindlicher Ideen durch Agitation und Propaganda ... «14

Eine derartige Betätigung kann man Seifert nicht vorwerfen. Er rief in seiner Rede nicht dazu auf, das Grundgesetz zu beseitigen, sondern dazu, es zu wahren und seine Intentionen zu erfüllen. Seifert hat seiner wissenschaftlichen Arbeit seit seiner Analyse der Spiegel-Affäre, besonders in der Notstandsauseinander-

$10 \mathrm{Vgl}$. Thieme, Deutsches Hochschuirecht, Berlin und Köln 1956, S. 64/s.

11 Werner Weber, Spannungen und Kräfte im westdeutschen Verfassungssystem, 3. Aufl., Berlin 1970, S. $34 / \mathrm{s}$.

12 Werner Weber, a. z. O., S. 32

13 Werner Weber, a. a. O., S. 26

$14 \mathrm{JZ}$ I971, S. 615, 6r9 mit ausführlichen Nachweisen. 
setzung, ein geradezu emphatisches Verfassungsverständnis zu Grunde gelegt. ${ }^{15}$ Er setzte sich für die Abwehr autoritärer Strömungen ein und forderte, aus den demokratischen und sozialstaatlichen Postulaten des Grundgesetzes die Konsequenzen zu ziehen. Auch in seiner Rede vom 25. I. I972 hat er nicht Kritik am Grundgesetz geübt, sondern an den gesellschaftlichen Verhältnissen, die die Realisierung der im Grundgesetz enthaltenen Ansprüche verhindern. Daß eine solche Position gegen die Pflicht zur Verfassungstreue verstößt, kann niemand ernstlich behaupten.

\section{Der Eingriff in die Autonomie der Hochschule}

Es bleibt, darauf hinzuweisen, daß im Zusammenhang mit dieser Affäre das Ministerium den Grundsatz der Autonomie der Hochschule angetastet hat.

Aus dem Grundrecht des Art. 5 Abs. 3 GG folgt das Recht der Hochschule auf Autonomie. Darauf berufen sich besonders die konservativen Kritiker der Hochschulreform. Die Universitäten sind nicht nachgeordnete Behörden der Kultusverwaltungen. Diese können in inneruniversitären Angelegenheiten nur im Wege der Rechtsaufsicht und nur in ganz bestimmtem, eng umgrenztem Umfang eingreifen.

Der Staatssekretär im niedersächsischen Kultusministerium hat vor dem teach-in am 25. Januar 1972 drei Professoren der hannoverschen Universität, darunter Seifert, ohne Angabe von Gründen durch dienstliche Weisungen in das Kultusministerium bestellt. Dort ermahnte er sie zur Zurückhaltung auf dem geplanten teach-in.

Der Staatssekretär hat damit versucht, inhaltlich in die Ausübung von Lehre und Forschung einzugreifen. Es gibt bisher wohl keinen Fall, daß ein Ministerialbeamter mit dienstrechtlichen Mitteln auf den Ablauf einer universitären Veranstaltung Einfluß nehmen wollte. Man stelle sich vor, daß diese Praxis Schule macht: Alle irgendwie der Ministerialbürokratie »verdächtigen « Hochschulangehörigen müßten sidh dann gefallen lassen, daß ihre Veranstaltungen von den Beamten des Kultusministeriums unter dem Hinweis auf mögliche disziplinarrechtliche Folgen zensiert werden.

Nach allgemeinem deutschem Hochschulrecht erledigt die Hochschule ihre Angelegenheiten selbst. Nur wenn sie dazu ausnahmsweise nicht in der Lage ist, darf das Ministerium eingreifen. Das Verwaltungsgericht Berlin hat im einzelnen dargelegt, unter welchen Voraussetzungen die Staatsaufsicht direkt in universitäre Angelegenheiten eingreifen kann: Sie muß zunächst der Hochschule einen ausreichenden Ermessensspielraum belassen. Nur dann besteht »die Notwendigkeit des Einschreitens der Staatsaufsicht, wenn die Universitätsorgane ihren Pflichten nicht nachkommen können oder wollen und insbesondere gegen verfassungsfeindliche und strafbare Handlungen ... nicht vorgehen. ${ }^{16} \mathrm{Daß}$ diese Voraussetzungen hier vorgelegen hätten, ist nicht festzustellen und auch nicht behauptet worden. Der Eingriff in die universitären Angelegenheiten war damit unzulässig. Er ist auch nicht mit der Ausübung der Disziplinarbefugnis zu rechtfertigen. Universitätsangehörige dürfen beamtenrechtlich nicht wie Angehörige eines Ministeriums behandelt werden.

15 Vgl. bes. sein Nachwort zu: Jürgen Seifert (Hg.), 20 Jahre Grundgesetz, Luchtcrhand Texte 26, Neuwied/Berlin 1969 .

$18 \mathrm{JZ} \times 97 \mathrm{r}, \mathrm{S} .6 \mathrm{x}, 6 \mathrm{xg} / 20$. 
Der Fall hat für alle Hochschulangehörigen exemplarische Bedeutung. Denn hier wird zum ersten Mal seit längerer Zeit wieder versucht, kritische Äußerungen eines Hochschullehrers in der Universität mit den Mitteln des Beamtenrechts zu unterbinden. Wenn dieser Versuchsballon eines Landesministeriums auch in anderen Bundesländern Schule machen sollte, dann müßten selbst bescheidene Ansätze einer gesellschaftskritisch-emanzipativen Wissenschaftspraxis aufgegeben werden. Der institutionalisierte Freiraum, den die Wissenschaftsfreiheit des Art. s Abs. 3 GG und die darin enthaltene Selbstverwaltungsgarantie der Hochschule gewährt, wäre zerschlagen. Forschung und Lehre wären von den politischen Vorurteilen der Ministerialbürokratie eingekesselt.

Zwar ist eine derartige Disziplinierung von Hochschulangehörigen mit der noch herrschenden Meinung im Hochschulrecht nicht vereinbar. Wie der Fall zeigt, sind aber auch anerkannte Rechtsgrundsätze keine Garantie dagegen, daß nicht Versuche gemacht werden, das Hochschulrecht im Interesse der Gegenreform zu funktionalisieren.

Es besteht die Gefahr, daß die Freiheit von Forschung und Lehre und der Grundsatz von der Selbstverwaltung der Hochschule nur noch von den Konservativen zur Abwehr der Hochschulreform in Anspruch genommen werden, daß diese Freiheiten aber denjenigen zunehmend verweigert werden, die in der Universität eine emanzipative Forschungs- und Lehrpraxis intendieren. Unter diesen Umständen ist es politisch wichtig, die im herkömmlichen Hochschulrecht enthaltenen Freiheitsgarantien zu verteidigen.

Alexander v. Brünneck

\section{Revolutionäre Gewalt und RAF*}

"Die Kommunisten haben die Gewalt nicht erfunden, sondern vorgefunden." Dieser Satz des französischen Philosophen Merleau-Ponty stellt das Problem der revolutionären Gewalt auf die Füße. Allgemein ausgedrückt setzt die marxistische Auffassung revolutionärer Gewalt die bestimmte historische Analyse einer politischen Situation voraus, aus der die militärische Strategie und Taktik als untergeordnetes Moment der politischen Strategie der Revolution entwickelt wird. Lenin hat nach der Revolution von I 905 die Kriterien für ein bewaffnetes Eingreifen der revolutionären Kräfte formuliert:

„Erstens unterscheidet sich der Marxismus von allen primitiven Formen des Sozialismus dadurch, daß er die Bewegung nicht an irgendeine bestimmte Kampfform bindet. Er erkennt die verschiedensten Kampfformen an, und zwar serfindet er sie nicht, sondern faßt nur die im Verlauf der Bewegung von selbst entstehenden Formen des Kampfes der revolutionären Klassen verallgemeinernd

* Oberarbeitete Fassung eines Diskussionsbeitrages auf dem teach-in gegen die Suspendierung von Peter Brückner am 25. 1. 1972 in Hannover. 